\title{
Light quality-dependent regulation of photoprotection and antioxidant properties in rice seedlings grown under different light-emitting diodes
}

\author{
L.H. TRAN ${ }^{\dagger}$ (ID), D.G. $\mathrm{LEE}^{\dagger}$, and S. JUNG ${ }^{+}$ \\ School of Life Sciences and Biotechnology, BK21 Plus KNU Creative Bioresearch Group, \\ Kyungpook National University, 41566 Daegu, Korea
}

\begin{abstract}
We examined light quality-dependent regulation of photoprotection and antioxidant properties in rice seedlings. Seedlings grown under red or green light-emitting diodes (LEDs) showed declines in quantum yield of electron transport through PSII, maximum relative electron transport rates, and photochemical quenching compared to the white and blue LED, but no difference in maximum photochemical efficiency of PSII was found. Blue LED treatment resulted in greater increases in not only nonphotochemical quenching, but also zeaxanthin formation, expressions of $\beta$-carotene hydroxylase and violaxanthin de-epoxidase, and anthocyanin compared to other LED treatments. By contrast, green and red LEDs increased activities of superoxide dismutase and ascorbate peroxidase as well as expression levels of catalase $(C A T) a$ and $C A T b$ to white and blue LEDs. Green and red LEDs also greatly upregulated expressions of phytochrome (PHY) A, PHYB, PHYC, and cryptochrome 2 compared to white and blue LEDs, whereas blue LED upregulated $C R Y 1$. Our results demonstrate that specific wavelengths of different LEDs differentially influence photosynthetic performance, photoprotection, and antioxidant properties, possibly through regulating expressions of phytochrome and cryptochrome genes in a light-quality-dependent manner.
\end{abstract}

Keywords: antioxidant enzyme; chlorophyll fluorescence quenching; xanthophyll.

\section{Introduction}

Light is not only an essential energy source for plants but also an important signal influencing plant development (Wu et al. 2007, Tran and Jung 2017, Yu et al. 2017). When more light is absorbed than utilized for photosynthesis, the excess excitation energy should be dissipated otherwise it is passed to molecular oxygen and reactive oxygen species (ROS) are generated. Therefore, photosynthesis could not exist without efficient photoprotective mechanisms against potential damage induced by unutilized light energy (Demmig-Adams et al. 2012, Wang et al. 2018). Excitation

\section{Highlights}

- Seedlings grown under blue LED ensure sufficient levels of photoprotection

- Seedlings grown under green and red LEDs increase activities of antioxidant enzymes

- Antioxidant enzyme activities show positive correlations to phytochrome expressions
Received 2 October 2020

Accepted 27 November 2020

Published online 15 December 2020

${ }^{+}$Corresponding author

e-mail: sjung@knu.ac.kr

phone: $+82-53-950-7364$

fax: $+82-53-943-6925$

Abbreviations: $\alpha$ - the initial slope of the light curve; APX - ascorbate peroxidase; BCH - $\beta$-carotene hydroxylase; Car - carotenoids; CAT - catalase; Chl - chlorophyll; CRY - cryptochrome; FM - fresh mass; $F_{v} / F_{m}$ - maximum photochemical efficiency of PSII; $\mathrm{I}_{\mathrm{k}}$ - irradiance at the onset of light saturation; LED - light-emitting diode; NPQ - nonphotochemical quenching; PHY - phytochrome; POD - peroxidase; $\mathrm{q}_{\mathrm{P}}$ - photochemical quenching; $\mathrm{RCL}$ - rapid light curve; $\mathrm{rETR}_{\max }$ - maximum relative electron transport rate; ROS - reactive oxygen species; SOD - superoxide dismutase; VDE - violaxanthin de-epoxidase; $\Phi_{\mathrm{PSII}}$ - quantum yield of electron transport through PSII.

Acknowledgment: This research was supported by Basic Science Research Program through the National Research Foundation of Korea (NRF) funded by the Ministry of Education (NRF-2018R1D1A1B07043814).

These authors contributed equally to the article.

Conflict of interest: The authors declare that they have no conflict of interest. 
energy in excess can be removed by de-excitation of excited singlet chlorophyll $\left({ }^{1} \mathrm{Chl}^{*}\right)$ (dissipation) and deexcitation of reactive forms of oxygen (antioxidation) (Demmig-Adams et al. 2012).

Under excess light conditions, light-harvesting complexes (LHCs) perform photoprotective quenching functions to prevent harmful chemical species such as triplet chlorophyll $\left({ }^{3} \mathrm{Chl} *\right)$ and singlet oxygen $\left({ }^{1} \mathrm{O}_{2}\right)$ from forming and damaging the photosynthetic apparatus (DemmigAdams et al. 2012). Chl $a$ fluorescence is used as an indicator of photosynthetic energy conversion, and a decrease in fluorescence as a result of light energy being converted into chemical energy is called photochemical quenching ( $\mathrm{q}_{\mathrm{P}}$ ) (Murchie and Lawson 2013, Foyer et al. 2017). The $\mathrm{q}_{\mathrm{p}}$ indicates not only the capacity of open PSII reaction centers and the excited energy used in photosynthesis but also reflects the stress induced by excess energy excitation on PSII. Nonphotochemical quenching (NPQ) is a process in which excess absorbed light energy is dissipated into heat, and it is a photoprotection mechanism against highlight stress (Ruban 2016).

Carotenoids (Car) have been known as the nonenzymatic antioxidants to protect the photosynthetic apparatus, and photoprotection by the dissipation of excess light energy is aided by the xanthophyll cycle carotenoids (Gilmore and Yamamoto 1991, Demmig-Adams et al. 2012, Jahns and Holzwarth 2012). Violaxanthin deepoxidase (VDE) converts violaxanthin to zeaxanthin via the intermediate antheraxanthin, thus sustaining dissipation of excess excitation energy as heat at PSII (Jahns and Holzwarth 2012). The other photoprotective pigments, anthocyanins, function as a light attenuator to protect the photochemical apparatus against excess light (Chalker-Scott 1999, Nakabayashi et al. 2014). While thermal dissipation of energy is useful in preventing the formation of ROS, additional photoprotective mechanisms exist to detoxify ROS. Antioxidation of harmful ROS generated under excess light acts as a sink for unutilized electrons (Demmig-Adams et al. 2012). ROS are generated when electrons not utilized in photosynthesis are donated to $\mathrm{O}_{2}$, thus creating superoxide $\left(\mathrm{O}_{2}{ }^{\cdot-}\right)$ that can be converted to hydrogen peroxide $\left(\mathrm{H}_{2} \mathrm{O}_{2}\right)$ by superoxide dismutase (SOD). Ascorbate peroxidase (APX), peroxidase (POD), and catalase (CAT) subsequently detoxify $\mathrm{H}_{2} \mathrm{O}_{2}$ (DemmigAdams et al. 2012, Foyer and Noctor 2013).

In higher plants, specific wavelengths of light play important roles in the regulation of growth, development of photosynthetic apparatus, Chl formation, and stomatal opening (Wu et al. 2007, Yu et al. 2017). Phytochromes are red/far-red light receptors that regulate photomorphogenesis, including de-etiolation and plant shape formation (Wang and Deng 2003). Cryptochromes are the bluelight photoreceptors that mediate inhibition of hypocotyl elongation, enhancement of cotyledon expansion, anthocyanin accumulation, and stomatal opening (Zhang et al. 2018). Also, cryptochromes regulate blue light-dependent biosynthesis of ROS (Consentino et al. 2015, El-Esawi et al. 2017) and are considered as key regulators of a range of plant stress responses, such as high light, heat, drought, and salinity (Ma et al. 2016, D'Amico-Damião and Carvalho 2018). The green light has discrete effects on plant biology (Folta and Maruhnich 2007), and the mechanisms that sense this light quality are now being elucidated.

Light-emitting diodes (LEDs) are characterized by their narrow spectrum and emerging as a promising tool for greenhouse crop production (Mitchell et al. 2012). Many studies have shown the effects of light intensities on photoinhibition of PSII (Jung et al. 2000, Hakala et al. 2005, Kouřil et al. 2013) and light quality on antioxidant capacity (Wu 2016, Yu et al. 2017). However, the effects of light quality on photoprotection and enzymatic antioxidant systems, particularly with the involvement of photoreceptors in these processes, are not well characterized yet. In our previous study, rice seedlings grown under white and blue LEDs were stronger, as indicated by greater leaf area and shoot biomass, than those of green and red LEDs, showing that light qualities influence the growth characteristics of seedlings (Tran and Jung 2017).

In the present study, we examined the influence of light spectral quality on photosynthetic performance and energy dissipation by analyzing photosynthetic efficiency and fluorescence quenching in rice seedlings grown under different LED wavelengths. To assess photoprotection and antioxidant properties under different LEDs, we determined the activities and gene expression of ROS-scavenging enzymes as well as the contents of photoprotective pigments. We also analyzed expression profiles of phytochrome and cryptochrome genes to characterize the involvement of photoreceptors in the modulation of energy dissipation and antioxidation.

\section{Materials and methods}

Plant growth and light treatment conditions: Rice (Oryza sativa cv. Dongjin) seeds were germinated at $25^{\circ} \mathrm{C}$ under the dark conditions for $5 \mathrm{~d}$ before irradiation. Then, the rice seedlings were grown under various types of lightemitting diodes (Union LED Electronic Co., Siheung-si, Korea) for $10 \mathrm{~d}$. Each LED treatment was conducted in separately controlled chambers to be free from spectral interference among treatments. Fifteen pots were placed in each LED chamber and seven plants were grown in each pot which was filled with commercial greenhouse compost. The LED array chambers were programmed to provide a 14-h-light/10-h-dark photoperiod at PPFD of approximately $150 \mu \mathrm{mol}$ (photon) $\mathrm{m}^{-2} \mathrm{~s}^{-1}$. The rice seedlings were grown under four different light sources with broadspectrum white LED (420-680 nm) as a control, blue LED (460-490 nm), green LED (520-550 nm), and red LED $(620-650 \mathrm{~nm})$ conditions. The youngest, fully developed leaves of rice seedlings were sampled $4 \mathrm{~h}$ after illumination $(11: 00 \mathrm{~h})$ on the last day of LED treatments.

Measurement of Chl $\boldsymbol{a}$ fluorescence: Chl $\boldsymbol{a}$ fluorescence was measured in vivo using a pulse amplitude modulation fluorometer (JUNIOR-PAM, Walz, Effeltrich, Germany) after dark adaptation for $30 \mathrm{~min}$. The minimum fluorescence $\left(\mathrm{F}_{0}\right)$ at open PSII centers was determined by measuring 
light, while the maximum fluorescence $\left(\mathrm{F}_{\mathrm{m}}\right)$ at closed PSII centers was examined after an application of a 0.8 -s pulse of saturating light $\left[3,500 \mu \mathrm{mol}\left(\right.\right.$ photon) $\left.\mathrm{m}^{-2} \mathrm{~s}^{-1}\right]$. Actinic light $\left[150 \mu \mathrm{mol}\right.$ (photon) $\mathrm{m}^{-2} \mathrm{~s}^{-1}$, growth light source for each LED treatment] was applied to measure the steadystate $\mathrm{Chl}$ fluorescence $\left(\mathrm{F}_{\mathrm{s}}\right)$. In the light-adapted state, $\mathrm{F}_{\mathrm{m}}{ }^{\prime}$ was measured by applying a saturating pulse, while $\mathrm{F}_{0}{ }^{\prime}$ was measured by switching off the actinic light for $2 \mathrm{~s}$ after the saturating pulse and applying far-red light. The ratio of $F_{v}$ to $F_{m}$, representing the activity of PSII, was used to assess the functional damage to the plants. The quantum yield of electron transport through PSII $\left(\Phi_{\mathrm{PSII}}=\Delta \mathrm{F} / \mathrm{F}_{\mathrm{m}}{ }^{\prime}\right)$ was calculated according to Genty et al. (1989). Coefficient of photochemical fluorescence quenching $\left(\mathrm{q}_{\mathrm{P}}\right)$ was used to estimate the fraction of open PSII centers and calculated as follows: $\mathrm{q}_{\mathrm{P}}=\left(\mathrm{F}_{\mathrm{m}}{ }^{\prime}-\mathrm{F}^{\prime}\right) /\left(\mathrm{F}_{\mathrm{m}}{ }^{\prime}-\mathrm{F}_{0}{ }^{\prime}\right)$, where $\mathrm{F}_{0}{ }^{\prime}$, the minimal $\mathrm{Chl}$ fluorescence yield of a preilluminated sample; $\mathrm{F}^{\prime}$, the level of the fluorescence curve during treatments and shortly before a saturating pulse is applied; $F_{m}{ }^{\prime}$, maximal Chl fluorescence in the light-adapted state (Schreiber et al. 1986). NPQ was also quantified, as previously done by Bilger and Björkman (1990) according to the Stern-Volmer equation, $\mathrm{NPQ}=\mathrm{F}_{\mathrm{m}} / \mathrm{F}_{\mathrm{m}}{ }^{\prime}-1$.

The rapid light curve (RLC) was also determined with a gradual increase of irradiance in 12 steps with 10 -s intervals ranging from 0 to $1,500 \mu \mathrm{mol}$ (photon) $\mathrm{m}^{-2} \mathrm{~s}^{-1}$ using blue light of PAM instrument as actinic light for RLC analysis. The parameters of the RLCs, including maximum relative ETR $\left(\mathrm{rETR}_{\max }\right)$, the efficiency of electron transport ( $\alpha$, the initial slope of the light curve), and the light saturation $\mathrm{I}_{\mathrm{k}}$ (irradiance at the onset of light saturation), were calculated following the Platt's formula (Jassby and Platt 1976, Harrison and Platt 1986).

Protein extraction, SDS-PAGE, and immunoblot analysis: Total protein was extracted by grinding frozen leaf material in a buffer containing $2 \%(\mathrm{w} / \mathrm{v})$ SDS, $56 \mathrm{mM}$ $\mathrm{Na}_{2} \mathrm{CO}_{3}, 12 \%$ (w/v) sucrose, $56 \mathrm{mM}$ dithiothreitol (DTT), and 2 mM EDTA, pH 8.0, followed by sonication. Total soluble proteins were collected by centrifugation at $12,000 \times g$ for $20 \mathrm{~min}$ and used for gel blot analysis. The proteins were separated on a $14 \%$ SDS-PAGE and electroblotted onto PVDF membranes. Immunodetection was performed using standard procedures (Roche, Basel, Switzerland). The polyclonal antibodies against lightharvesting chlorophyll-binding proteins of PSI (Lhca) and PSII (Lhcb) and $\alpha$-tubulin were purchased from Agrisera.

Determination of carotenoids (Car): For Car analysis, leaf tissues were ground in $100 \%$ acetone containing $10 \mathrm{mg}$ of $\mathrm{CaCO}_{3}$. The extracts were centrifuged at $16,000 \times g$ for $10 \mathrm{~min}$ and the resulting supernatants were collected. The pigments were separated by HPLC as previously described by Gilmore and Yamamoto (1991) using Prominence HPLC system (Shimadzu, Kyoto, Japan) equipped with SPD-M20A Prominence Photodiode Array Detector (Shimadzu). A Spherisorb ODS-1 column (5- $\mu \mathrm{m}$ particle size, $250 \mathrm{~mm} \times 4.6 \mathrm{~mm}$ inner diameter) was obtained from Waters (Milford, MA, USA). Solvent A (acetonitrile:methanol:Tris- $\mathrm{HCl}$ buffer $0.1 \mathrm{M}, \mathrm{pH}$ 8.0,
$72: 8: 3, \mathrm{v} / \mathrm{v} / \mathrm{v}$ ) was run isocratically from 0 to $4 \mathrm{~min}$ followed by a 2.5 -min linear gradient to $100 \%$ solvent B (methanol:hexane, 4:1, v/v) at flow rate of $2 \mathrm{ml} \mathrm{min}^{-1}$. HPLC runs were carried out at $445 \mathrm{~nm}$ for xanthophylls and $\beta$-carotene. Specific extinction coefficients (E1\% $\left.1 \mathrm{~cm}^{-1}\right)$ used for quantification were lutein (2,550 at 445 $\mathrm{nm})$, zeaxanthin $(2,540$ at $450 \mathrm{~nm})$, antheraxanthin $(2,350$ at $446 \mathrm{~nm})$, violaxanthin $(2,550$ at $443 \mathrm{~nm})$, neoxanthin $(2,243$ at $439 \mathrm{~nm})$, and $\beta$-carotene $(2,590$ at $450 \mathrm{~nm})$.

Determination of anthocyanin: Anthocyanins were extracted by homogenizing leaves $[0.1 \mathrm{~g}$ of fresh mass $(\mathrm{FM})]$ in a precooled mortar with $1 \mathrm{ml}$ of acidified $(1 \%$ $\mathrm{HCl})$ methanol and maintained at $4{ }^{\circ} \mathrm{C}$ for $4 \mathrm{~h}$ to avoid the degradation of $\mathrm{Chl}$ whose products may interfere with the absorption of anthocyanins at $530 \mathrm{~nm}$ (peak absorption of anthocyanin) and $657 \mathrm{~nm}$ (peak absorption of Chl degradation products) (Mancinelli et al. 1975). Particles were removed by centrifugation at $10,000 \times g$ for $30 \mathrm{~min}$. Anthocyanins were calculated by using $\mathrm{A}_{530}-$ $0.25 \mathrm{~A}_{657}$ to account for the contribution by $\mathrm{Chl}$. Anthocyanin content was calculated as cyanidin-3glucoside using $29,600 \mathrm{M}^{-1} \mathrm{~cm}^{-1}$ as molecular extinction coefficient and 445 as molecular mass.

Assays for antioxidant enzymes: Leaves ( 0.25 g of FM) were macerated to a fine powder in a mortar under liquid $\mathrm{N}_{2}$. Soluble proteins were extracted by homogenizing the powder in $2 \mathrm{ml}$ of $100 \mathrm{mM}$ potassium phosphate buffer, $\mathrm{pH} 7.5$, containing 2 mM EDTA, 1\% PVP-40, and $1 \mathrm{mM}$ phenylmethylsulfonyl fluoride. Insoluble material was removed by centrifugation at $15,000 \times g$ for $20 \mathrm{~min}$ at $4^{\circ} \mathrm{C}$. As for maintenance of consistent catalase (CAT, EC 1.11.1.6) electrophoretic mobility was found to require the presence of DTT, an aliquot of each sample was made to $10 \mathrm{mM}$ DTT to be used for the CAT zymogram. Equal amounts of protein were electrophoresed on $10 \%$ nondenaturing polyacrylamide gels at $4^{\circ} \mathrm{C}$ for $1.5 \mathrm{~h}$ at a constant current of $30 \mathrm{~mA}$. Following separation of ascorbate peroxidase (APX, EC 1.11.1.11), gels were soaked in $50 \mathrm{mM}$ potassium phosphate buffer, $\mathrm{pH} 7.0$, containing $2 \mathrm{mM}$ ascorbate for $30 \mathrm{~min}$ (Rao et al. 1996). The gels were incubated in the same buffer containing $4 \mathrm{mM}$ ascorbate and $2 \mathrm{mM} \mathrm{H}_{2} \mathrm{O}_{2}$ for $20 \mathrm{~min}$ and then soaked in $50 \mathrm{mM}$ potassium phosphate buffer, $\mathrm{pH} 7.8$, containing $28 \mathrm{mM}$ tetramethyl ethylenediamine and $2.45 \mathrm{mM}$ nitroblue tetrazolium (NBT) for $15 \mathrm{~min}$. The CAT activity was detected by incubating the gels in $3.27 \mathrm{mM} \mathrm{H}_{2} \mathrm{O}_{2}$ for $25 \mathrm{~min}$, rinsing them in water, and staining them in a solution of $1 \%$ potassium ferricyanide and $1 \%$ ferric chloride for $4 \mathrm{~min}$ (Woodbury et al. 1971). The staining of peroxidase (POD, EC 1.11.1.7) isozymes was achieved by incubating gels in sodium citrate buffer (pH 5.0) containing $9.25 \mathrm{mM} p$-phenylenediamine and $3.92 \mathrm{mM} \mathrm{H}_{2} \mathrm{O}_{2}$ for $15 \mathrm{~min}$ (Olson and Varner 1993). Gels were stained for superoxide dismutase (SOD, EC 1.15.1.1) isoforms by soaking in $50 \mathrm{mM}$ potassium phosphate $(\mathrm{pH}$ 7.8) containing $2.5 \mathrm{mM} \mathrm{NBT}$ in darkness for $25 \mathrm{~min}$, followed by soaking in $50 \mathrm{mM}$ potassium phosphate ( $\mathrm{pH} 7.8$ ) containing $28 \mathrm{mM}$ NBT and $28 \mu \mathrm{M}$ riboflavin in 
darkness for $30 \mathrm{~min}$. The gels were then exposed to light for 30 min (Rao et al. 1996).

APX activity was measured spectrophotometrically (UV-2550, Shimadzu, Japan) by monitoring the decline in $\mathrm{A}_{290}$ as ascorbate was oxidized (Chen and Asada 1989). The 3-ml reaction volume contained $100 \mathrm{mM}$ potassium phosphate buffer ( $\mathrm{pH} 7.5$ ), $0.5 \mathrm{mM}$ ascorbate, and $0.2 \mathrm{mM} \mathrm{H}_{2} \mathrm{O}_{2}$ at $25^{\circ} \mathrm{C}$. Total CAT activity was performed spectrophotometrically in a $3 \mathrm{ml}$ volume containing $50 \mathrm{mM}$ potassium phosphate buffer, $\mathrm{pH} 7.0$, containing $20 \mathrm{mM}$ $\mathrm{H}_{2} \mathrm{O}_{2}$ by monitoring $\mathrm{H}_{2} \mathrm{O}_{2}$ destruction at $240 \mathrm{~nm}$ (Beers and Sizer 1952). POD activity was determined specifically with guaiacol at $470 \mathrm{~nm}$ (Egley et al. 1983). The reaction mixture contained $40 \mathrm{mM}$ potassium phosphate buffer (pH 6.9), $1.5 \mathrm{mM}$ guaiacol, and $6.5 \mathrm{mM} \mathrm{H}_{2} \mathrm{O}_{2}$ in a $3-\mathrm{ml}$ volume. For APX, CAT, and POD, enzyme activities were expressed as $\mu \mathrm{mol} \mathrm{g}^{-1}(\mathrm{FM}) \mathrm{min}^{-1}$. SOD activity was determined using the xanthine oxidase-cytochrome $c$ method at pH 10.0 (Baum and Scandalios 1981). The assay was performed at $25^{\circ} \mathrm{C}$ in a $3-\mathrm{ml}$ volume containing $50 \mathrm{mM}$ sodium carbonate buffer ( $\mathrm{pH} 10.0), 0.1 \mathrm{mM}$ EDTA, $15 \mu \mathrm{M}$ ferricytochrome $c$, and $0.05 \mathrm{mM}$ xanthine. SOD activity was expressed as units $\mathrm{g}^{-1}(\mathrm{FM})$.

RNA extraction and qRT-PCR: Total RNA was prepared from leaf tissues using TRIzol Reagent (Invitrogen, Waltham, MA, USA), and $5 \mu \mathrm{g}$ of RNA from each sample was used for the reverse transcription reaction (ImProm-II ${ }^{\mathrm{TM}}$ Reverse Transcription System, Promega, WI, USA). Subsequently, $50 \mathrm{ng}$ of cDNA was used for qRT-PCR analysis. The qRT-PCR analysis was carried out using the StepOnePlus Real-Time PCR System (Applied Biosystems, Waltham, MA, USA) using Power SYBR Green PCR Master Mix (Applied Biosystems) and genespecific primers (Table 1S, supplement). The qRT-PCR program consisted of $2 \mathrm{~min}$ at $50^{\circ} \mathrm{C}, 10 \mathrm{~min}$ at $95^{\circ} \mathrm{C}$, and 40 cycles of $15 \mathrm{~s}$ at $95^{\circ} \mathrm{C}$, and $1 \mathrm{~min}$ at $60^{\circ} \mathrm{C}$. A melting curve analysis was performed after every PCR reaction to confirm the accuracy of each amplified product. All reactions were set up in triplicate. The sample of white LED treatment was used as the calibrator, with the expression level of the sample set to 1. Actin was used as an internal control.

Statistical analysis: Data were expressed as means \pm SE. Differences were analyzed by Duncan's multiple range test. $p$-values $<0.05$ were considered to be significant. Correlation analysis was also performed using the SPSS software (SPSS Inc., Chicago, IL, USA).

\section{Results}

Photosynthetic efficiency and photoprotective quenching mechanisms: The 5-d-old etiolated rice seedlings were exposed to white, blue, green, or red LED light in cycles of 14-h light and 10-h dark for $10 \mathrm{~d}$. In all seedlings grown under different LEDs, the youngest, fully developed leaves did not develop any necrosis. Although seedlings were grown under a moderate PPFD light intensity of $150 \mu \mathrm{mol}\left(\right.$ photon) $\mathrm{m}^{-2} \mathrm{~s}^{-1}$, necrotic spots, which are characteristics of photodamage, often appeared on older leaves of seedlings grown under green or red LED (data not shown). Seedlings grown under green and red LEDs also exhibited reductions in surface area of the youngest, fully developed leaves and dry shoot biomass compared to those of white and blue LEDs, while seedlings grown under blue LED exhibited the shortest shoot height and a significant increase in leaf width (Tran and Jung 2017). We supposed that a narrow spectrum of green and red LEDs may impose a lower PSII excitation pressure on whole seedlings compared to blue and broad-spectrum white LEDs, reducing shoot biomass. Thus, we examined how seedlings treated with different wavelengths of LEDs develop differential photoprotective mechanisms to optimize their growth.

For rapid estimation of photosynthetic characteristics and quenching mechanisms under different LED light qualities, we measured $\mathrm{Chl}$ fluorescence parameters in the youngest, fully developed leaves. There was no difference in $F_{v} / F_{m}$, a trait positively correlated with the vitality of PSII, of seedlings grown under monochrome blue, green, or red LED compared to broad-spectrum white LED (Fig. 1A). Seedlings grown in green or red LED showed declines in $\Phi_{\text {PSII }}$, which is the quantum yield of electron transport through PSII, compared to white and blue LEDs (Fig. 1B).

Leaf photosynthetic capacity was also assessed by the RLC approach. The relative electron transfer rate of PSII (rETR) rapidly increased with increasing light intensity (Fig. 1E). The rETR in red and green LED treatments reached a steady state faster, while white and blue LED treatments reached the steady state at higher light levels. The maximum electron transport rate of PSII, $\mathrm{rETR}_{\max }$, and the light-saturation point, $I_{k}$, in seedlings under white and blue LED treatments were significantly greater than those of green and red LEDs (Table 1). The initial slope of the light response, $\alpha$, was lower in green and red LEDs than that of white and blue LEDs, with the lowest value in green LED.

The $\mathrm{q}_{\mathrm{p}}$ is used to indicate the onset of photoinhibition because a linear relationship was observed between $\mathrm{q}_{\mathrm{P}}$ and photoinhibition of PSII (van Wijk and van Hasselt 1993). Seedlings grown in red and green LEDs showed lower $\mathrm{q}_{\mathrm{P}}$ values as compared to white and blue LEDs (Fig. 1C). Nonradiative energy dissipation through NPQ was higher by $44 \%$ in blue LED treatment, compared to that of white LED treatment (Fig. 1D). In comparison to white LED, NPQ levels were lower by 60 and 54\% in seedlings under green and red LEDs, respectively (Fig. 1D). Next, we evaluated the organization of the photosynthetic apparatus in seedlings under respective light irradiations of LEDs by quantitatively analyzing Lhca and Lhcb proteins of PSI and PSII, respectively. Expression levels of Lhca and Lhcb proteins, which are major components of light-harvesting antennae, were not different after all LED treatments (Fig. 1F).

Photoprotective pigments and expression of genes involved in zeaxanthin biosynthesis: To assess the effects of light quality on photoprotective pigments, we 


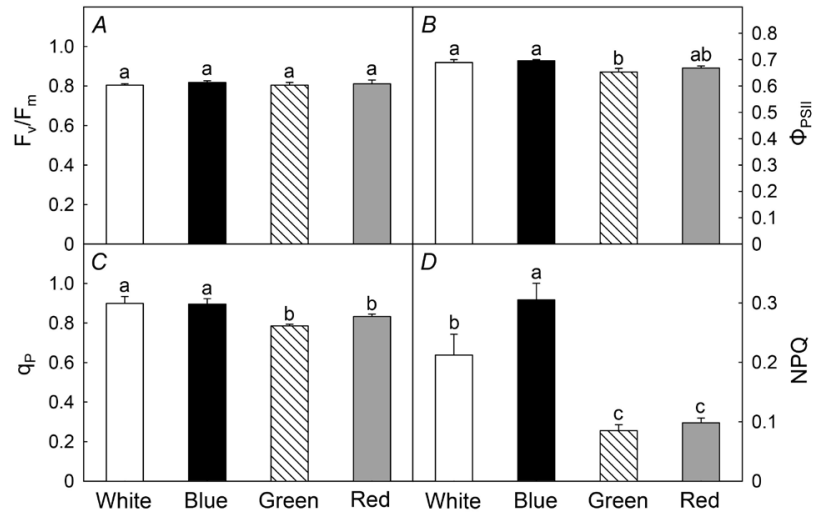

E
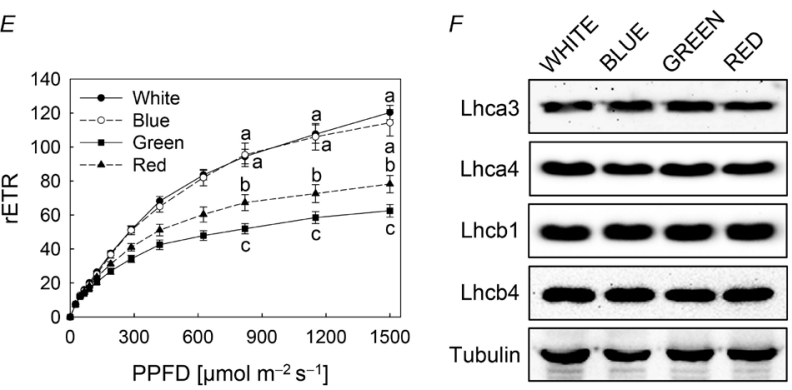

Fig. 1. Effects of different wavelengths of LEDs on function and structure of photosystems and quenching parameters in rice seedlings. (A) $\mathrm{F}_{\mathrm{v}} / \mathrm{F}_{\mathrm{m}}$, efficiency of excitation capture by open PSII reaction centers. $(B) \Phi_{\text {PSII }}$, quantum yield of electron transport through PSII. (C) $\mathrm{q}_{\mathrm{P}}$, photochemical quenching, (D) NPQ, nonphotochemical quenching. (E) Rapid light curves (RLCs) of the relative electron transport rate (rETR). ( $F$ ) Immunoblot analysis of the light-harvesting chlorophyll-binding proteins of PSI (Lhca3 and Lhca4) and PSII (Lhcb1 and Lhcb4). Alphatubulin was used as a loading control. The 5-d-old etiolated rice seedlings were exposed to different light-emitting diodes (LEDs) with a 14-h-light/10-h-dark photoperiod for $10 \mathrm{~d}$. White - broad spectrum-white LED (420-680 nm) as a control; Blue - blue LED (460-490 nm); Green - green LED (520-550 nm); Red red LED $(620-650 \mathrm{~nm})$. The data represent the mean \pm SE of nine replicates from three independent experiments. Means denoted by the same letter did not differ significantly at $P<0.05$ according to Duncan's multiple range test.

examined the contents of carotenoids and anthocyanins. The contents of lutein and $\beta$-carotene, which are potent ROS scavengers, in seedlings treated with white and red LEDs were higher than those of blue and green LEDs (Table 2). In seedlings grown under blue LED, contents of zeaxanthin, an important component of the xanthophyll cycle, increased by $65 \%$ compared to white LED and were higher than those of green and red LEDs by 253 and $283 \%$, respectively (Table 2). Contents of antheraxanthin, violaxanthin, and neoxanthin as well as the sum of violaxanthin, antheraxanthin, and zeaxanthin (VAZ) were not significantly different between various LED treatments (Table 2). The de-epoxidation state of xanthophyll cycle pigments, which is expressed as $[(\mathrm{Z}+0.5 \mathrm{~A}) / \mathrm{VAZ}]$, exhibited higher values in white and blue LEDs compared to those in green and red LEDs. In comparison to white
Table 1. Effects of different wavelengths of LEDs on maximum relative ETR ( $\mathrm{rETR}_{\max }$, the efficiency of electron transport ( $\alpha$, the initial slope of the light curve), and the light saturation $I_{k}$ (irradiance at the onset of light saturation). The plants were subjected to the same treatments as in Fig. 1, and treatment notations are the same as in Fig. 1. The data represent the mean \pm $\mathrm{SE}$ of nine replicates from three independent experiments. Within each column, means denoted by the same letter did not differ significantly at $P<0.05$ according to Duncan's multiple range test.

\begin{tabular}{lcll}
\hline Treatment & $\mathrm{rETR}_{\max }$ & $\alpha$ & $\mathrm{I}_{\mathrm{k}}\left[\mu \mathrm{mol} \mathrm{m}{ }^{-2} \mathrm{~s}^{-1}\right]$ \\
\hline White & $115.7 \pm 5.2^{\mathrm{a}}$ & $0.187 \pm 0.007^{\mathrm{a}}$ & $621.9 \pm 24.4^{\mathrm{a}}$ \\
Blue & $112.3 \pm 10.5^{\mathrm{a}}$ & $0.178 \pm 0.006^{\mathrm{a}}$ & $635.3 \pm 58.9^{\mathrm{a}}$ \\
Green & $56.1 \pm 3.3^{\mathrm{b}}$ & $0.147 \pm 0.014^{\mathrm{b}}$ & $398.7 \pm 30.7^{\mathrm{b}}$ \\
Red & $74.5 \pm 5.7^{\mathrm{b}}$ & $0.171 \pm 0.011^{\mathrm{ab}}$ & $454.5 \pm 48.8^{\mathrm{b}}$ \\
\hline
\end{tabular}

LED, anthocyanin contents were the highest in seedlings grown under blue LED and lowest in seedlings grown under white and green LEDs (Table 2).

We used qRT-PCR to analyze the effect of different light wavelengths on the expression of genes involved in zeaxanthin formation. In comparison to white LED treatment, the expression level of $B C H$ encoding $\beta$-carotene hydroxylase $(\mathrm{BCH})$, which catalyzes the hydroxylation reaction from $\beta$-carotene to zeaxanthin, was about 2 -fold upregulated in blue LED treatment (Fig. 2A). Seedlings grown under blue LED also upregulated expression of $V D E$ encoding $\mathrm{VDE}$, which catalyzes the conversion from violaxanthin to zeaxanthin, compared to white LED (Fig. 2B). Expression levels of $B C H$ and $V D E$ in seedlings treated with green and red LEDs were similar to those of white LED.

Activity and gene expression of ROS-scavenging enzymes: We monitored changes in isozyme profiles and activities of ROS-scavenging enzymes in response to different LED wavelengths. Total SOD activities were higher in seedlings grown under green and red LEDs compared to white and red LEDs, with a greater increase in green LED (Fig. 3A). These were mainly due to the elevated activity of SOD isozymes 2 and 3 in seedlings grown under green LED, and isoform 3 in the case of red LED (Fig. 3E). Although the total CAT activities did not differ between different LEDs (Fig. $3 B$ ), the staining activities of CAT isozymes 1 and 2 greatly increased in green and red LED treatments and decreased in blue LED treatment (Fig. 3F) as compared to white LED. The total POD activity was lower in blue LED, but higher in red LED than that of white LED (Fig. 3C). In a comparison of isoforms, seedlings grown under blue LED exhibited lower activities of all POD isozymes compared to white LED (Fig. 3G). POD isozyme 2 decreased in green LED treatment compared to white LED, whereas POD isozymes 3 and 4 increased in green and red LED treatments. Marked increases in both APX-staining activities and total APX activities were observed in seedlings grown under green or red LED, with a greater increase in green LED (Fig. 3D,H). 


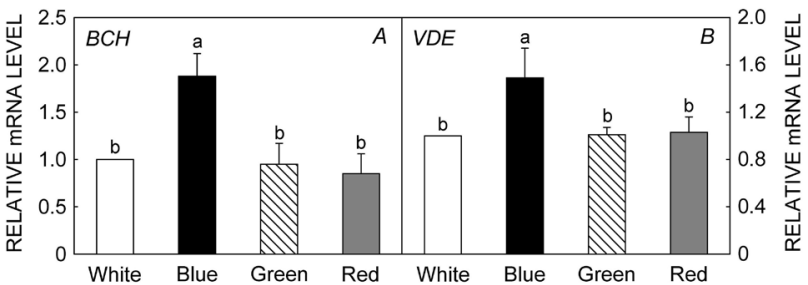

Fig. 2. Expression of genes involved in zeaxanthin formation in rice seedlings grown under different wavelengths of LEDs. (A) $\beta$-carotene hydroxylase $(B C H)$. (B) Violaxanthin de-epoxidase $(V D E)$. The plants were subjected to the same treatments as in Fig. 1. Treatment notations are the same as in Fig. 1. Total RNAs were purified from plants and reverse transcribed. The resulting cDNAs were used as templates for qRT-PCR using Actin as an internal control. The white LED (control) was used for normalization, with the expression level of the sample set to 1 . The data represent the mean $\pm \mathrm{SE}$ of nine replicates from three independent experiments. Means denoted by the same letter did not differ significantly at $P<0.05$ according to Duncan's multiple range test.

We next examined whether the transcript levels of genes encoding $\mathrm{H}_{2} \mathrm{O}_{2}$-scavenging enzymes, CAT and APX, directly correspond to their enzyme activities under different wavelengths of LEDs. Expression of $C A T a$ exhibited 2.2- and 3.1-fold upregulation in seedlings treated with green and red LEDs, respectively, compared to white LED treatment, but downregulation in blue LED (Fig. 4A). Seedlings grown under blue, green, or red LED exhibited increases in transcript levels of $C A T b$ compared to white LED, with greater increases in green and red LEDs (Fig. 4B). However, the transcript level of $C A T c$ was highest in seedlings treated with blue LED compared to other LEDs (Fig. 4C). In comparison to white LED, transcript levels of $A P X a$ increased only in green LED, whereas transcript levels of $A P X b$ increased in blue, green, and red LEDs (Fig. 4D,E). CAT activity showed positive correlations to $C A T a$ and $C A T b$ with Pearson's correlation coefficients of 0.897 and 0.537 , respectively, but a negative correlation to CATc with Pearson's correlation coefficient of -0.712 (Table 3). APX activity showed positive correlations to $A P X a$ and $A P X b$ with Pearson's correlation coefficients of 0.763 and 0.705 , respectively.

Expression profiles of phytochrome and cryptochrome genes: To assess the possible role of photoreceptors in photoprotection and antioxidant properties under different wavelengths of LEDs, we monitored the expression of genes encoding the apoproteins of phytochromes and cryptochromes, which are red/far-red light and blue light photoreceptors, respectively. In comparison to broadspectrum white LED, seedlings grown under green or red LED greatly upregulated $P H Y A, P H Y B$, and $P H Y C$ genes encoding phytochrome $\mathrm{A}, \mathrm{B}$, and $\mathrm{C}$, with greater increases of $P H Y A$ and $P H Y B$ in red LED (Fig. 5A-C). Transcript levels of $P H Y A, P H Y B$, and $P H Y C$ in seedlings treated with blue LED were not significantly different from those of white LED. 

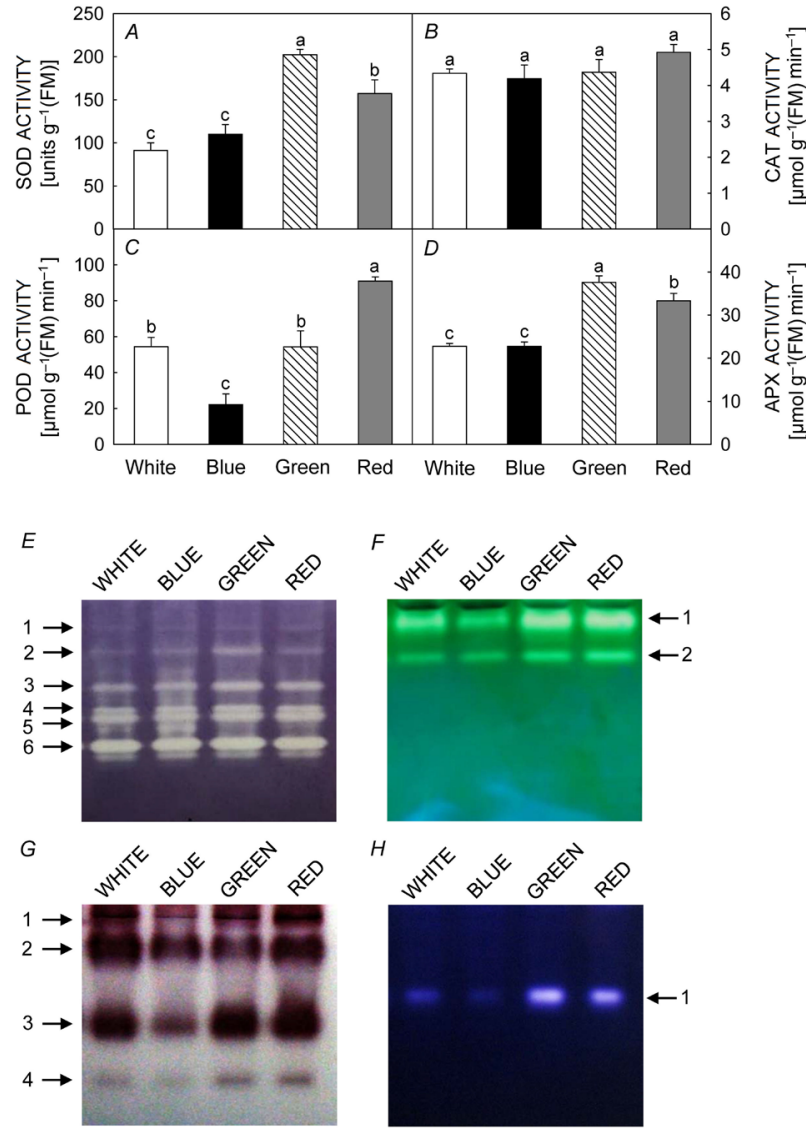

Fig. 3. Isozyme profiles and activities of ROS-scavenging enzymes in rice seedlings grown under different wavelengths of LEDs. $(A, E)$ Superoxide dismutase (SOD); $(B, F)$ catalase (CAT); $(C, G)$ peroxidase (POD); $(D, H)$ ascorbate peroxidase (APX). The plants were subjected to the same treatments as in Fig. 1. Treatment notations are the same as in Fig. 1. The numbers indicate each isozyme of antioxidant enzymes in order of detected bands from the top. The data represent the mean \pm SE of nine replicates from three independent experiments. Means denoted by the same letter did not differ significantly at $P<0.05$ according to Duncan's multiple range test.

In seedlings grown under blue LED, the expression level of CRY1, a gene encoding cryptochrome 1, increased by $78 \%$ compared to white LED (Fig. 5D), whereas the expression level of $C R Y 2$ was similar to that of white LED. Expression levels of CRY1 did not differ significantly between white, green, and red LED treatments, whereas expression levels of $C R Y 2$ were upregulated by 85 and $53 \%$ in green and red LED treatments, respectively, compared to white LED (Fig. 5D,E). These results demonstrate that different wavelengths of LEDs greatly influence expression profiles of phytochrome and cryptochrome genes.

Correlation between antioxidant enzyme activity and expression of photoreceptor genes: Pearson's correlation coefficient was applied to evaluate the relationship between antioxidant enzyme activity and expression of photoreceptor genes. The enzyme activities of SOD, CAT,

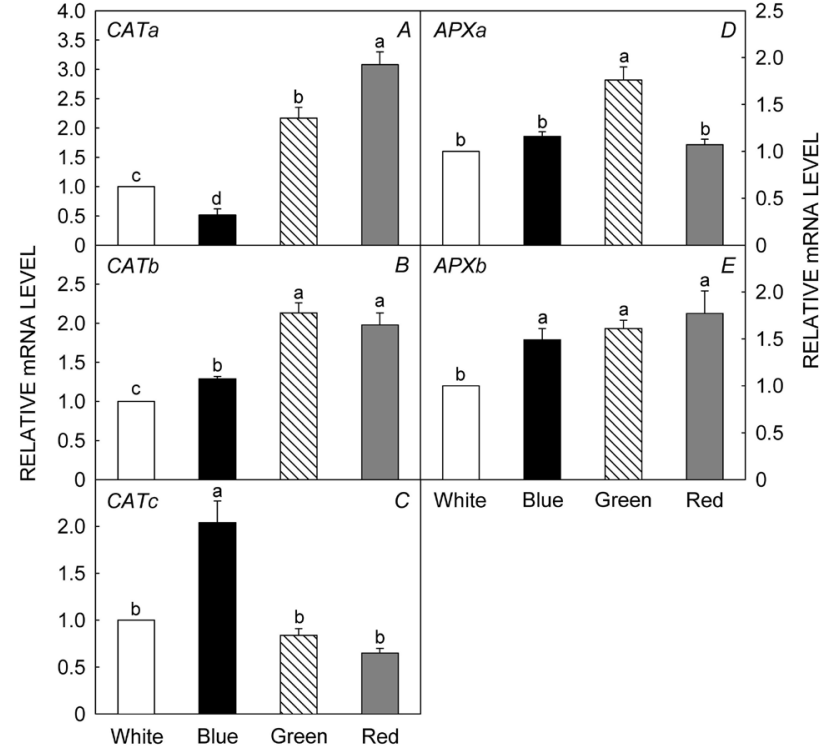

Fig. 4. Effects of different wavelengths of LEDs on the expression of genes encoding the $\mathrm{H}_{2} \mathrm{O}_{2}$-scavenging enzymes, catalase (CAT) $(A-C)$ and ascorbate peroxidase $(A P X)(D, E)$. The plants were subjected to the same treatments as in Fig. 1. Treatment notations are the same as in Fig. 1. Total RNAs were purified from plants and reverse transcribed. The resultant cDNAs were used as templates for qRT-PCR using Actin as an internal control. The white LED (control) was used for normalization, with the expression level of the sample set to 1 . The data represent the mean \pm SE of nine replicates from three independent experiments. Means denoted by the same letter did not differ significantly at $P<0.05$ according to Duncan's multiple range test.

POD, and APX showed positive correlations to PHYA, PHYB, and PHYC (Table 3). Among these, total APX activity showed a significant positive correlation to $P H Y C$ with Pearson's correlation coefficient of 0.946 . Negative correlations between CRY1 vs. enzyme activities of SOD, CAT, POD, and APX were observed, with a significant coefficient of -0.931 between $C R Y 1$ and POD activity. By contrast, $C R Y 2$ showed a significant positive correlation to SOD and APX activities.

Expression levels of $P H Y A, P H Y B$, and $P H Y C$ showed positive correlations to $C A T a, C A T b$, and $A P X b$, with significant correlations to $C A T a$ and $C A T b$, whereas negative correlations between $C A T c$ vs. expression levels of PHYA, PHYB, and PHYC were found (Table 3). Negative correlations between $C R Y 1$ vs. expression levels of $C A T a$ and $C A T b$ were observed, whereas $C R Y 1$ showed a significant positive correlation to $C A T c$ with a coefficient of 0.995. CRY2 showed positive correlations to CATa, $C A T b, A P X a$, and $A P X b$ with Pearson's correlation coefficients of $0.748,0.969,0.833$, and 0.728 , respectively. Overall, the activities of SOD, CAT, POD, and APX as well as the expression levels of $C A T a, C A T b$, and $A P X b$ showed positive correlations to $P H Y A, P H Y B$, and $P H Y C$. On the other hand, $C R Y 1$ and $C R Y 2$ exhibited negative and positive correlations, respectively, to the activities and expression of some antioxidant enzymes. 


\section{Discussion}

Specific wavelengths of different LEDs greatly influence not only growth characteristics (Tran and Jung 2017), but also photoprotection and antioxidation mechanisms in rice seedlings. When rice seedlings were exposed to different wavelengths of LEDs, levels of $F_{v} / F_{m}$, as well as Lhca and Lhcb proteins in blue, red, and green LEDs were the same as in white LED (Fig. $1 A, F$ ), indicating no noticeable damage of photosynthetic apparatus. However, seedlings grown in

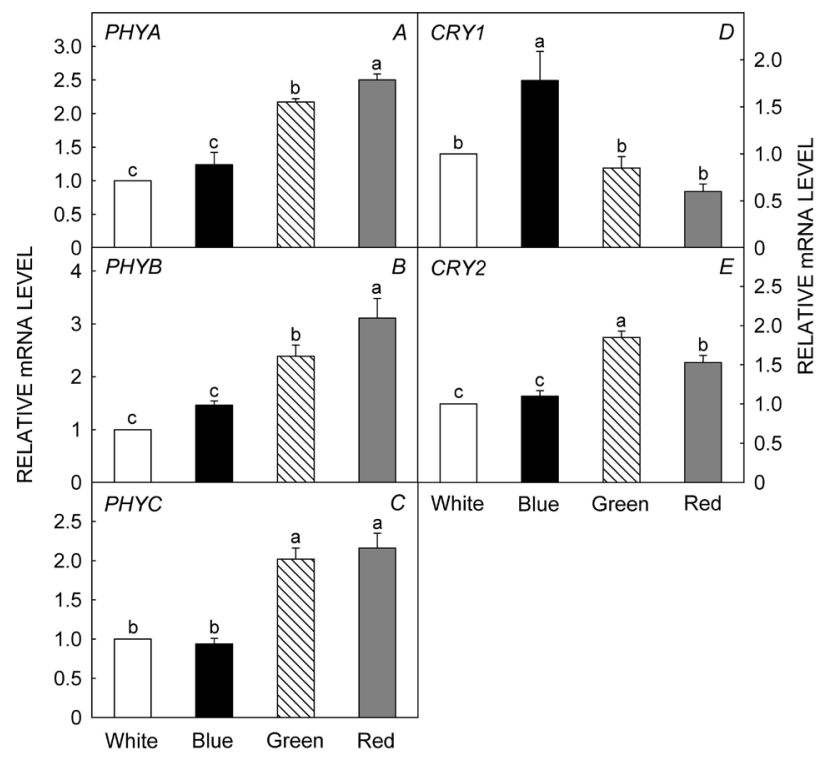

Fig. 5. Effects of different wavelengths of LEDs on the expression of genes encoding photoreceptors, phytochromes $(P H Y)(A-C)$ and cryptochromes $(C R Y)(D, E)$. The plants were subjected to the same treatments as in Fig. 1. Treatment notations are the same as in Fig. 1. Total RNAs were purified from plants and reverse transcribed. The resultant cDNAs were used as templates for qRT-PCR using Actin as an internal control. The white LED (control) was used for normalization, with the expression level of the sample set to 1 . The data represent the mean \pm SE of nine replicates from three independent experiments. Means denoted by the same letter did not differ significantly at $P<0.05$ according to Duncan's multiple range test. red or green LED showed low efficiency of photochemical utilization of absorbed light energy, as indicated by the decrease of $\Phi_{\text {PSII }}, \mathrm{rETR}_{\max }, \alpha$, and $\mathrm{I}_{\mathrm{k}}$, compared to seedlings grown in white or blue LED (Fig. 1, Table 1). These results imply the negative effects of monochromatic green and red LED on the photosynthetic performance. Since Chls have light absorption peaks at blue and red wavelengths, light energy absorbed by Chls is likely to be associated with photodamage to PSII (Zavafer et al. 2015). In comparison to white and blue LEDs, Chl contents of green and red LEDs were lower, while $\mathrm{Chl} a / b$ higher in blue, red, and green LEDs than that in white LED, indicating that structure of photosynthetic apparatus is altered depending on the light quality, as indicated previously by Tran and Jung (2017). The wavelength of blue light is known as particularly effective photoinhibition (He et al. 2015). However, rice seedlings treated with blue LED showed higher $\Phi_{\text {PSII }}$, RLC parameters, and $\mathrm{q}_{\mathrm{P}}$ compared to monochrome green and red LEDs (Fig. 1), which may explain the greatest shoot biomass among different monochrome LEDs (Tran and Jung 2017).

The $\mathrm{q}_{\mathrm{P}}$, that is the relative reduction state of $\mathrm{Q}_{\mathrm{A}}$, reflects the fraction of open PSII reaction centers (Murchie and Lawson 2013). The high $\mathrm{q}_{\mathrm{P}}$ value in white and blue LEDs (Fig. 1C) indicates that more absorbed light energy was consumed by photochemical processes in seedlings grown under white or blue light. By contrast, the low $\mathrm{q}_{\mathrm{p}}$ value in seedlings grown under green or red LED implies a decrease in the proportion of available excitation energy used for photochemistry. NPQ, a protective process, increased by $44 \%$ in blue LED treatment compared to white LED treatment (Fig. 1D). The significant increase of NPQ in blue LED indicates the increased thermal dissipation of excess excitation energy in PSII, which is the first line of defense in photoprotection. In seedlings under green and red LEDs, NPQ levels significantly decreased compared to white LED (Fig. 1D), whereas the red alga Pyropia haitanensis under red light showed the highest NPQ compared to white, blue, and green light (Wu 2016).

Zeaxanthin enhances thermal dissipation of excess absorbed light energy, which is measured as NPQ (Demmig-Adams et al. 2012, Jahns and Holzwarth 2012). Zeaxanthin is synthesized by either conversion

Table 3. Pearson's correlation coefficients of antioxidant enzyme activity $v s$. the expression of photoreceptor genes. ${ }^{*},{ }^{* *}-$ correlation is significant at $P<0.05$ and $P<0.01$, respectively. APX - ascorbate peroxidase; CAT - catalase; CRY - cryptochrome; PHY - phytochrome; POD - peroxidase; SOD - superoxide dismutase.

\begin{tabular}{lccccccc}
\hline Parameter & PHYA & PHYB & PHYC & CRY1 & CRY2 & CAT & APX \\
\hline SOD & 0.845 & 0.768 & 0.872 & -0.521 & $0.998^{* *}$ & - & - \\
CAT & 0.781 & 0.824 & 0.768 & -0.792 & 0.408 & - & - \\
POD & 0.723 & 0.729 & 0.769 & $-0.931^{*}$ & 0.436 & - & - \\
APX & 0.904 & 0.832 & $0.946^{*}$ & -0.688 & $0.989^{* *}$ & - & - \\
CATa & $0.944^{*}$ & $0.933^{*}$ & $0.963^{* *}$ & -0.873 & 0.748 & 0.897 & - \\
CATb & $0.951^{*}$ & 0.908 & $0.944^{*}$ & -0.567 & $0.969^{* *}$ & 0.537 & - \\
CATc & -0.634 & -0.574 & -0.755 & $0.995^{* *}$ & -0.565 & -0.712 & - \\
APXa & 0.446 & 0.327 & 0.514 & -0.220 & 0.833 & - & 0.763 \\
APXb & 0.875 & 0.904 & 0.773 & -0.257 & 0.728 & - & 0.705 \\
\hline
\end{tabular}


from $\beta$-carotene or de-epoxidation from violaxanthin (Demmig-Adams et al. 2012, Jahns and Holzwarth 2012). In addition to low $\beta$-carotene content, expression levels of $B C H$ and $V D E$ were greater in blue LED treatment than those of white, green, and red LED treatments (Table 2, Fig. 2), showing that the great accumulation of zeaxanthin can be explained by transcriptional regulation of zeaxanthin biosynthetic genes, with more conversion from $\beta$-carotene. Anthocyanin is another pigment that has some protection for the chloroplasts against excess radiation (Chalker-Scott 1999, Zhang et al. 2016). Among different LEDs, the highest anthocyanin contents in seedlings grown under blue LED (Table 1) were similar to the findings that anthocyanin contents in grape and strawberry treated with blue wavelength were higher than those with red wavelength (Kondo et al. 2014, Zhang et al. 2018). Our results demonstrate that rice seedlings grown under different light qualities produce differential contents of photoprotective pigments, particularly with high accumulations of zeaxanthin and anthocyanin under blue LED. Although anthocyanins are not involved in the plastidic energy dissipation processes mediated by NPQ, the accumulation of anthocyanins may provide an additional means of photoprotection by acting as a light screen.

ROS depend on not only nonenzymatic antioxidants including Car but also enzymatic antioxidants (Suzuki et al. 2012, Noctor et al. 2014). Increased activities of SOD in seedlings grown under green or red LED (Fig. 3A,E) indicated the increased conversion of $\mathrm{O}_{2}{ }^{-}$into $\mathrm{H}_{2} \mathrm{O}_{2}$. Upregulations of $C A T a$ and $C A T b$ in seedlings treated with green and red LEDs were correlated with the increased staining activities of CAT, but not with total CAT activities (Figs. 3, 4). Red light also significantly increased CAT and SOD activities compared to other light sources in $P$. haitanensis (Wu 2016). In comparison to white, green, and red LEDs, staining and total activities of POD decreased under blue LED treatment (Fig. 3C, $G$ ). $A P X a$ was upregulated only in green LED, whereas $A P X b$ was upregulated in blue, green, and red LEDs compared to white LED (Fig. 4D,E). It is assumed that both transcriptional upregulation of $A P X$ and the direct effect of specific light wavelengths on APX enzyme can be the basis for the marked increase in APX activity under green and red LEDs (Fig. 3D,H). Among different LED lights, blue LED light caused low demand for antioxidant enzymes, i.e., the low activities of POD and APX, which is likely due to sufficient energy dissipation through zeaxanthin and NPQ. By contrast, the lack of dissipative processes in green and red LED treatments may necessitate increased demand for SOD and APX.

Photoreceptor-mediated light signaling plays a pivotal role in photomorphogenesis (Neff and Chory 1998, Wang and Deng 2003, Zhang et al. 2018), but its role in photoprotection especially against excess excitation energy needs further clarification. Rice seedlings grown under green or red LED significantly upregulated $P H Y A$, $P H Y B$, and $P H Y C$ compared to broad-spectrum white LED (Fig. 5A-C). Exposure of etiolated Avena seedlings to redlight pulse also showed a higher phytochrome transcript level than continuous white light (Colbert et al. 1985). The green light-mediated upregulations of the three $P H Y$ s (Fig. 5A-C) support the idea that phytochromes can absorb some green light (Folta and Maruhnich 2007). The high expression levels of the three PHY genes were positively correlated with the increased activities of SOD, CAT, and APX (Figs. 3, 5). Moreover, activities of SOD, CAT, POD, and APX as well as expressions of $C A T a, C A T b$, and $A P X b$ showed positive correlations to $P H Y A, P H Y B$, and $P H Y C$ (Table 3 ). These results suggest a possible role of phytochromes in light quality-dependent regulation of antioxidant enzymes. Sharma et al. (1976) has already observed in maize that the transformation of Pr into Pfr by absorption of red light increased POD activity. In phy $A$, phy $B$, phy $A B$, and crylphy $A B 1$ mutants, deficiencies in phytochromes and cryptochrome 1 affected the levels of antioxidant enzymes in response to UV stress or high light (Rusaczonek et al. 2015, Kreslavski et al. 2020). The expression patterns of $P H Y_{\mathrm{s}}$ in blue LED treatment were similar to those of white LED treatment (Fig. $5 \mathrm{~A}-\mathrm{C}$ ) indicated that besides red and far-red light, phytochromes have been shown to act as blue-light photoreceptors (Mohr 1994, Neff and Chory 1998).

To assess the involvement of cryptochromes in differential photoprotection under different wavelengths of LEDs, we examined expression profiles of $C R Y_{\mathrm{s}}$. In comparison to the white LED, the expression level of CRY1 significantly increased in seedlings grown under blue LED, which was followed by a higher content of anthocyanins (Table 1, Fig. 5D). CRY1 protein contents also increased severalfold on irradiation of Brassica napus seedlings with blue light (Chatterjee et al. 2006). On the other hand, expression levels of CRY2 were upregulated in green and red LED treatments, compared to white LED (Fig. 5E). The upregulation of CRY2 in red LED treatment could be explained by the fact that CRY is a functionally important blue and red light-activated flavoprotein (Beel et al. 2012). Besides, cryptochromes are known as greenlight receptors (Folta and Maruhnich 2007), and the green-absorbing state of CRY1 and CRY2 reverses blue light-induced responses (Banerjee et al. 2007, Bouly et al. 2007) such as the degradation of CRY2 (Li et al. 2011). Photolabile CRY2 is rapidly degraded in blue light, while CRY1 is a relatively stable photoreceptor (Ahmad et al. 1998). Among different LED treatments, the highest expression level of $C R Y 2$ in green LED treatment (Fig. 5E) may be related to green light-induced prevention of CRY2 degradation. Also, $C R Y 1$ and $C R Y 2$ exhibited negative and positive correlations, respectively, to the activities and expression of some antioxidant enzymes (Table 3), however, the correlations were weaker than those of PHYs. Kreslavski et al (2020) suggests that phytochromes together with CRY1 control the expression of $A P X$ genes under high-intensity light.

Conclusions: Our results demonstrate that specific wavelengths of different LEDs greatly influence photosynthetic performance, photoprotection, and antioxidant properties in rice seedlings. Seedlings grown under blue LED ensured sufficient levels of photoprotection by not only high NPQ, 
but also great increases in zeaxanthin, expressions of $B C H$ and $V D E$, and anthocyanin. Also, blue light-induced upregulation of CRY1 indicates that CRY1 is involved in energy dissipation mechanisms, thereby optimizing photosynthetic efficiency and photoprotection of PSII. By contrast, in seedlings grown under green and red LEDs, low levels of NPQ, zeaxanthin, and anthocyanin may necessitate increased activities of SOD and APX. The activities and expressions of antioxidant enzymes showed positive correlations to the expression of $P H Y$ genes, suggesting a possible role of phytochromes in the regulation of antioxidant enzymes. Our study provides more insight into light quality-dependent regulation of photoprotection and antioxidation mechanisms and their relationship with the expressions of phytochrome and cryptochrome genes.

\section{References}

Ahmad M., Jarillo J.A., Cashmore A.R.: Chimeric proteins between cry1 and cry 2 Arabidopsis blue light photoreceptors indicate overlapping functions and varying protein stability. Plant Cell 10: 197-207, 1998.

Banerjee R., Schleicher E., Meier S. et al:: The signaling state of Arabidopsis cryptochrome 2 contains flavin semiquinone. J. Biol. Chem. 282: 14916-14922, 2007.

Baum J.A., Scandalios J.G.: Isolation and characterization of the cytosolic and mitochondrial superoxide dismutases of maize. - Arch. Biochem. Biophys. 206: 249-264, 1981.

Beel B., Prager K., Spexard M. et al.: A flavin binding cryptochrome photoreceptor responds to both blue and red light in Chlamydomonas reinhardtii. - Plant Cell 24: 29923008, 2012.

Beers R.F., Sizer I.W.: A spectrophotometric method for measuring the breakdown of hydrogen peroxide by catalase. J. Biol. Chem. 195: 133-140, 1952.

Bilger W., Björkman O.: Role of the xanthophyll cycle in photoprotection elucidated by measurements of light-induced absorbance changes, fluorescence and photosynthesis in leaves of Hedera canariensis. - Photosynth. Res. 25: 173$185,1990$.

Bouly J.-P., Schleicher E., Dionisio-Sese M. et al.: Cryptochrome blue light photoreceptors are activated through interconversion of flavin redox states. - J. Biol. Chem. 282: 9383-9391, 2007.

Chalker-Scott L.: Environmental significance of anthocyanins in plant stress responses. - Photochem. Photobiol. 70: 1-9, 1999.

Chatterjee M., Sharma P., Khurana J.P.: Cryptochrome 1 from Brassica napus is up-regulated by blue light and controls hypocotyl/stem growth and anthocyanin accumulation. Plant Physiol. 141: 61-74, 2006.

Chen G.-X., Asada K.: Ascorbate peroxidase in tea leaves: Occurrence of two isozymes and the differences in their enzymatic and molecular properties. - Plant Cell Physiol. 30: 987-998, 1989.

Colbert J.T., Hershey H.P., Quail P.H.: Phytochrome regulation of phytochrome mRNA abundance. - Plant Mol. Biol. 5: 91-101, 1985.

Consentino L., Lambert S., Martino C. et al:: Blue-light dependent reactive oxygen species formation by Arabidopsis cryptochrome may define a novel evolutionarily conserved signaling mechanism. - New Phytol. 206: 1450-1462, 2015.

D'Amico-Damião V., Carvalho R.F.: Cryptochrome-related abiotic stress responses in plants. - Front. Plant Sci. 9: 1897, 2018.
Demmig-Adams B., Cohu C.M., Adams III W.W.: Dealing with the hazards of harnessing sunlight. - Nature Education 4: 18, 2012.

Egley G.H., Paul R.N., Vaughn K.C., Duke S.O.: Role of peroxidase in the development of water-impermeable seed coats in Sida spinosa L. - Planta 157: 224-232, 1983.

El-Esawi M., Arthaut L.-D., Jourdan N. et al:: Blue-light induced biosynthesis of ROS contributes to the signaling mechanism of Arabidopsis cryptochrome. - Sci. Rep.-UK 7: 13875, 2017.

Folta K.M., Maruhnich S.A.: Green light: a signal to slow down or stop. - J. Exp. Bot. 58: 3099-3111, 2007.

Foyer C.H., Noctor G.: Redox signaling in plants. - Antioxid. Redox Sign. 18: 2087-2090, 2013.

Foyer C.H., Ruban A.V., Noctor G.: Viewing oxidative stress through the lens of oxidative signalling rather than damage. Biochem. J. 474: 877-883, 2017.

Genty B., Briantais J.-M., Baker N.R.: The relationship between the quantum yield of photosynthetic electron transport and quenching of chlorophyll fluorescence. - BBA-Gen. Subjects 990: 87-92, 1989.

Gilmore A.M., Yamamoto H.Y.: Zeaxanthin formation and energy-dependent fluorescence quenching in pea chloroplasts under artificially mediated linear and cyclic electron transport. - Plant Physiol. 96: 635-643, 1991.

Hakala M., Tuominen I., Keränen M. et al.: Evidence for the role of the oxygen-evolving manganese complex in photoinhibition of Photosystem II. - BBA-Bioenergetics 1706: 68-80, 2005.

Harrison W.G., Platt T.: Photosynthesis-irradiance relationships in polar and temperate phytoplankton populations. - Polar Biol. 5: 153-164, 1986.

He J., Yang W., Qin L. et al.: Photoinactivation of photosystem II in wild-type and chlorophyll $b$-less barley leaves: which mechanism dominates depends on experimental circumstances. - Photosynth. Res. 126: 399-407, 2015.

Jahns P., Holzwarth A.R.: The role of the xanthophyll cycle and of lutein in photoprotection of photosystem II. BBA-Bioenergetics 1817: 182-193, 2012.

Jassby A.D., Platt T.: Mathematical formulation of the relationship between photosynthesis and light for phytoplankton. Limnol. Oceanogr. 21: 540-547, 1976.

Jung S., Kim J.S., Cho K.Y. et al:: Antioxidant responses of cucumber (Cucumis sativus) to photoinhibition and oxidative stress induced by norflurazon under high and low PPFDs. Plant Sci. 153: 145-154, 2000.

Kondo S., Tomiyama H., Rodyoung A. et al.: Abscisic acid metabolism and anthocyanin synthesis in grape skin are affected by light emitting diode (LED) irradiation at night. J. Plant Physiol. 171: 823-829, 2014.

Kouřil R., Wientjes E., Bultema J.B. et al.: High-light vs. low-light: Effect of light acclimation on photosystem II composition and organization in Arabidopsis thaliana. BBA-Bioenergetics 1827: 411-419, 2013.

Kreslavski V.D., Strokina V.V., Pashkovskiy P.P. et al.: Deficiencies in phytochromes A and B and cryptochrome 1 affect the resistance of the photosynthetic apparatus to high-intensity light in Solanum lycopersicum. - J. Photoch. Photobio. B 210: 111976, 2020.

Li X., Wang Q., Yu X. et al.: Arabidopsis cryptochrome 2 (CRY2) functions by the photoactivation mechanism distinct from the tryptophan (trp) triad-dependent photoreduction. P. Natl. Acad. Sci. USA 108: 20844-20849, 2011.

Ma D., Li X., Guo Y. et al.: Cryptochrome 1 interacts with PIF4 to regulate high temperature-mediated hypocotyl elongation in response to blue light. - P. Natl. Acad. Sci. USA 113: 224-229, 2016.

Mancinelli A.L., Yang C.-P.H., Lindquist P. et al.: Photocontrol 
of anthocyanin synthesis. III. The action of streptomycin on the synthesis of chlorophyll and anthocyanin. - Plant Physiol. 55: 251-257, 1975.

Mitchell C., Both A.J., Bourget C. et al.: LEDs: The future of greenhouse lighting. - Chron. Horticult. 52: 6-12, 2012.

Mohr H.: Coaction between pigment systems. - In: Kendrick R.E., Kronenberg G.H.M. (ed.): Photomorphogenesis in Plants. $2^{\text {nd }}$ Edition. Pp. 353-373. Springer, Dordrecht 1994.

Murchie E.H., Lawson T.: Chlorophyll fluorescence analysis: a guide to good practice and understanding some new applications. - J. Exp. Bot. 64: 3983-3998, 2013.

Nakabayashi R., Yonekura-Sakakibara K., Urano K. et al.: Enhancement of oxidative and drought tolerance in Arabidopsis by overaccumulation of antioxidant flavonoids. Plant J. 77: 367-379, 2014.

Neff M.M., Chory J.: Genetic interactions between phytochrome A, phytochrome B, and cryptochrome 1 during Arabidopsis development. - Plant Physiol. 118: 27-35, 1998.

Noctor G., Mhamdi A., Foyer C.H.: The roles of reactive oxygen metabolism in drought: Not so cut and dried. - Plant Physiol. 164: 1636-1648, 2014.

Olson P.D., Varner J.E.: Hydrogen peroxide and lignification. Plant J. 4: 887-892, 1993.

Rao M.V., Paliyath G., Ormrod D.P.: Ultraviolet-B- and ozoneinduced biochemical changes in antioxidant enzymes of Arabidopsis thaliana. - Plant Physiol. 110: 125-136, 1996.

Ruban A.V.: Nonphotochemical chlorophyll fluorescence quenching: Mechanism and effectiveness in protecting plants from photodamage. - Plant Physiol. 170: 1903-1916, 2016.

Rusaczonek A., Czarnocka W., Kacprzak S. et al.: Role of phytochromes A and B in the regulation of cell death and acclimatory responses to UV stress in Arabidopsis thaliana. J. Exp. Bot. 66: 6679-6695, 2015.

Schreiber U., Schliwa U., Bilger W.: Continuous recording of photochemical and non-photochemical chlorophyll fluorescence quenching with a new type of modulation fluorometer. - Photosynth. Res. 10: 51-62, 1986.

Sharma R., Sopory S.K., Guha-Mukherjee S.: Phytochrome regulation of peroxidase activity in maize. - Plant Sci. Lett. 6: $69-75,1976$

Suzuki N., Koussevitzky S., Mittler R., Miller G.: ROS and redox signalling in the response of plants to abiotic stress. Plant Cell Environ. 35: 259-270, 2012.

Tran L.H., Jung S.: Effects of light-emitting diode irradiation on growth characteristics and regulation of porphyrin biosynthesis in rice seedlings. - Int. J. Mol. Sci. 18: 641, 2017.

van Wijk K.J., van Hasselt P.R.: Photoinhibition of photosystem II in vivo is preceded by down-regulation through light-induced acidification of the lumen: Consequences for the mechanism of photoinhibition in vivo. - Planta 189: 359-368, 1993.

Wang F., Wu N., Zhang L. et al.: Light signaling-dependent regulation of photoinhibition and photoprotection in tomato. Plant Physiol. 176: 1311-1326, 2018.

Wang H., Deng X.W.: Dissecting the phytochrome A-dependent signaling network in higher plants. - Trends Plant Sci. 8: 172178, 2003.

Woodbury W., Spencer A.K., Stahmann M.A.: An improved procedure using ferricyanide for detecting catalase isozymes. Anal. Biochem. 44: 301-305, 1971.

Wu H.: Effect of different light qualities on growth, pigment content, chlorophyll fluorescence, and antioxidant enzyme activity in the red alga Pyropia haitanensis (Bangiales, Rhodophyta). - BioMed Res. Int. 2016: 7383918, 2016.

Wu M.-C., Hou C.Y., Jiang C.M. et al.: A novel approach of LED light radiation improves the antioxidant activity of pea seedlings. - Food Chem. 101: 1753-1758, 2007.

Yu W., Liu Y., Song L. et al.: Effect of differential light quality on morphology, photosynthesis, and antioxidant enzyme activity in Camptotheca acuminata seedlings. - J. Plant Growth Regul. 36: 148-160, 2017.

Zavafer A., Cheah M.H., Hillier W. et al.: Photodamage to the oxygen evolving complex of photosystem II by visible light. Sci. Rep.-UK 5: 16363, 2015.

Zhang T.-J., Chow W.S., Liu X.-T. et al.: A magic red coat on the surface of young leaves: anthocyanins distributed in trichome layer protect Castanopsis fissa leaves from photoinhibition. Tree Physiol. 36: 1296-1306, 2016.

Zhang Y., Jiang L., Li Y. et al.: Effect of red and blue light on anthocyanin accumulation and differential gene expression in strawberry (Fragaria $\times$ ananassa). - Molecules 23: 820, 2018.

(C) The authors. This is an open access article distributed under the terms of the Creative Commons BY-NC-ND Licence. 